\title{
DESIGUALDADES EN LA DIVISIÓN DEL TRABAJO EN FAMILIAS CON MENORES DURANTE EL CONFINAMIENTO POR COVID-19 EN ESPAÑA. ¿̇HACIA UNA MAYOR POLARIZACIÓN SOCIOECONÓMICA Y DE GÉNERO?!
}

Inequalities in the division of labor in families with children during COVID-19 confinement in Spain. Towards greater gender and socioeconomic polarization?

\author{
MARTA SEIZ \\ Universidad Nacional de Educación a Distancia \\ mseiz@poli.uned.es
}

Cómo citar/Citation Seiz, M. (2020).

Desigualdades en la división del trabajo en familias con menores durante el confinamiento por COVID-19 en España.

¿̇Hacia una mayor polarización socioeconómica y de género? lgualdadES, 3, 403-435.

doi: https://doi.org/10.18042/cepc/lgdES.3.05

(Recepción: 01/07/2020; aceptación tras revisión: 06/10/2020; publicación: 18/12/2020)

Resumen

El confinamiento de la población española en sus hogares y el cierre de los centros escolares y de cuidado debido a la pandemia de COVID-19 generaron un aumento inédito de demandas domésticas para las familias con menores, en tanto

1 Este artículo ha sido realizado gracias a la financiación de una ayuda Juan de La Cierva-Incorporación (IJC2018-038444-I) del Ministerio de Ciencia, Innovación y Universidades. 
que los progenitores tuvieron que adaptarse al teletrabajo o interrumpir su actividad laboral. Partiendo de una encuesta realizada durante el estado de alarma, se analiza si las familias en las que ambos mantuvieron su empleo recurriendo al teletrabajo y/o la flexibilidad horaria establecieron distribuciones de tareas más corresponsables que aquellas en las que no se dieron tales circunstancias. Los resultados muestran que este fue el caso, lo que sugiere una polarización social, basada en desigualdades de género, entre familias con y sin condiciones favorables al mantenimiento de un modelo igualitario de doble ingreso. El estudio también revela una mayor carga de trabajo para las mujeres durante el período analizado.

\title{
Palabras clave
}

División del trabajo; desigualdades sociales; igualdad de género; corresponsabilidad; cuidados; teletrabajo; flexibilidad; COVID-19.

\begin{abstract}
The confinement of the Spanish population within their households and the closure of schools and daycare centers due to the COVID-19 pandemic generated an unprecedented increase in domestic demands for families with children, as parents had to adapt to remote work or interrupt their labor market activity. Drawing on a survey carried out during the state of alarm, it is analyzed whether families where both parents maintained their jobs resorting to telework or flexibility established more co-responsible distributions of tasks than those where such circumstances where not present. Results show that such was the case, which suggests social polarization based on gender inequalities between families with and without favorable conditions for the maintenance of an egalitarian dual-earner model. The study also reveals a larger relative workload for women during the analyzed period.
\end{abstract}

\section{Keywords}

Division of labor; social inequalities; gender equality; housework; care; telework; flexibility; COVID-19. 


\section{SUMARIO}

I. INTRODUCCIÓN. II. CONTEXTO, MARCO TEÓRICO Y PREGUNTAS DE INVESTIGACIÓN. III. DATOS Y METODOLOGÍA. IV. RESULTADOS. V. CONCLUSIONES. BiblograFía. APÉNDICE.

\section{INTRODUCCIÓN}

A mediados de marzo de 2020, la inminente pandemia de COVID-19 situaba a la población mundial en una situación de emergencia, provocando en países de todos los continentes una profunda disrupción de la actividad social y económica. En el caso de España, el alcance y la velocidad de los contagios dieron lugar a la declaración de un estado de alarma y al decreto del confinamiento de la población. Durante la fase más restrictiva de este período, las familias se vieron obligadas a permanecer recluidas en sus hogares, sin posibilidad de contacto con personas ajenas a dicho núcleo, inclusive familiares. Los centros escolares, las escuelas infantiles y los centros de día se mantuvieron cerrados. Se produjo la interrupción de servicios no esenciales y del ejercicio de cualquier empleo que no pudiera llevarse a cabo de forma telemática desde el hogar. Esta situación generó, de la noche a la mañana, un incremento considerable de demandas de tiempo y trabajo a las que enfrentarse en el ámbito familiar. La permanencia forzosa de todos los miembros del hogar en el mismo a lo largo de la jornada, así como la ausencia de posibilidades de externalización, no podían sino generar un aumento de la cantidad e intensidad de las tareas domésticas y de cuidado, que habrían de ser asumidas exclusivamente en la esfera privada. En el caso de las familias con menores en edad escolar, se añadía la necesidad de ocuparse también parcialmente de su atención educativa, siguiendo los materiales e instrucciones proporcionados telemáticamente por los docentes. En estas circunstancias, y dadas las interrupciones de la actividad laboral o la transición al teletrabajo que la situación impuso en muchos hogares, se planteó un escenario inédito para la renegociación súbita de los roles familiares, en un contexto de vertiginosa reorganización de los tiempos de toda la sociedad.

Este artículo, basado en una encuesta realizada a través de internet en la fase más estricta del estado de alarma («Conciliación y usos del tiempo 
en España en el contexto de la pandemia COVID-19»), examina el resultado de las renegociaciones mencionadas con el fin de analizar el alcance de las diferencias de género en la división del trabajo durante el confinamiento. Se pone el foco en núcleos familiares biparentales con hijos menores de dieciocho años, por dos motivos: en primer lugar, la intensidad y variedad de las necesidades domésticas que debieron abordarse en paralelo en estos hogares fue considerable. En segundo lugar, en las generaciones con hijos de esta edad la participación laboral de los dos progenitores es relativamente frecuente (González y Jurado Guerrero, 2015), por lo que cabía esperar un cierto potencial de conflicto y redefinición de roles ante los constreñimientos impuestos por la crisis de la COVID-19.

El principal objetivo del estudio es examinar, partiendo de la teoría sobre la disponibilidad de tiempo, si el acceso a medidas de conciliación (teletrabajo y/o flexibilidad) por parte de ambos progenitores tuvo un impacto visible sobre la posibilidad de establecer divisiones del trabajo corresponsables durante el confinamiento. Parece razonable imaginar distribuciones de tareas más igualitarias en familias donde tanto el padre como la madre pudieron mantener su empleo mediante el trabajo en remoto, o bien turnándose en sus jornadas laborales. En una situación de intensas demandas domésticas y de cuidado, como las impuestas por el estado de alarma, debería resultar más fácil mantener una distribución equitativa del trabajo si ambos progenitores, además de compartir las responsabilidades económicas, tienen presencia conjunta en el hogar y pueden repartirse otras tareas, o si ambos pueden alternarse en su dedicación a las mismas. Por el contrario, la falta de acceso a las medidas mencionadas podría haber conducido a algunos progenitores - con mayor probabilidad las madres ${ }^{2}$ - a interrumpir o reducir notablemente su implicación laboral para ocuparse del trabajo no remunerado, con la consiguiente generación de cargas domésticas desiguales y un acceso diferencial a los recursos económicos. Como la pérdida de empleo derivada de la crisis de la COVID-19 fue muy significativa (Carta et al., 2020), otros hogares habrían tenido que afrontar una situación de desempleo o inactividad indeseada por parte de algún progenitor o de ambos, así como la necesidad de negociar nuevos acuerdos en un contexto de marcada incertidumbre y dificultad económica. Estas circunstancias podrían haber reforzado patrones de género tradicionales o haberlos contrarrestado en función de la reacción de cada

2 Las madres muestran, tanto en el contexto internacional como en el caso de España, una mayor propensión que los padres a abandonar el mercado de trabajo o reducir su participación laboral cuando surgen necesidades intensas de cuidado. Véase, por ejemplo, Grunow y Evertsson (2019). 
pareja a la situación y a la mayor disponibilidad de tiempo paterna. Como se explicará en la sección teórica, el grado de internalización por parte de las parejas de las normas sociales imperantes con respecto a los roles considerados adecuados para madres y padres es potencialmente clave en este sentido.

En resumen, el análisis pretende arrojar luz sobre posibles diferencias, en términos de igualdad de género en la división intrafamiliar del trabajo, entre parejas en las que ambos miembros pudieron hacer uso del teletrabajo y/o la flexibilidad laboral durante el estado de alarma, y aquellas en las que no se pudieron utilizar estos recursos. Se intenta asimismo desentrañar qué papel ha jugado cada una de estas medidas en el fomento de una distribución de tareas corresponsable. Del mismo modo, se presta atención a la posible relación de las tendencias encontradas con variables de estratificación social, en concreto con el nivel educativo y el tipo de ocupación de la madre. Estos dos factores podrían haber ejercido una influencia adicional importante sobre los arreglos familiares respecto a la organización y los usos del tiempo, ya que se asocian con la internalización de valores de género más o menos tradicionales (Farré y Vella, 2013) y con los recursos que las mujeres pueden movilizar en las negociaciones con sus parejas (Lundberg y Pollak, 1996).

En último término, este trabajo busca identificar si existen indicios de una polarización social incipiente desencadenada o agudizada por la crisis de la COVID-19 y basada en la magnitud de las desigualdades de género en las familias. La principal hipótesis que se plantea es que determinadas condiciones laborales - en concreto, el acceso a medidas de conciliación por parte de los dos progenitores durante el confinamiento- podrían haber facilitado, en una minoría de familias, una distribución corresponsable de tareas que no se habrá podido establecer en muchas otras. De ser el caso, este fenómeno podría tener a medio plazo una repercusión más amplia en términos de vulnerabilidad socioeconómica, especialmente en un contexto de recesión como el que se anticipa. La corresponsabilidad en el reparto de tareas y roles dentro de las parejas es un factor esencial para que mujeres y hombres puedan mantenerse igualmente activos en el mercado de trabajo y consolidar su posición económica (véase, por ejemplo, González y Jurado-Guerrero, 2015). En consecuencia, aquellas familias que hayan podido establecer durante el estado de alarma las bases de una división corresponsable del trabajo estarán mejor posicionadas para mantener dos empleos durante una crisis sin tener que reducir jornada e ingresos, y por tanto más protegidas frente al riesgo de pobreza. Por el contrario, en aquellas en las que se hayan reproducido patrones tradicionales y en las que la mujer haya interrumpido o limitado su participación laboral para cuidar o atender otras necesidades domésticas, no solo habrá tenido lugar un aumento de la vulnerabilidad inmediata del hogar, sino que podrían instaurarse dinámicas de comportamiento difíciles 
de romper. Este último escenario es una posibilidad real ante la perspectiva de elevadas tasas de desempleo como consecuencia de la pandemia, especialmente teniendo en cuenta que el empleo femenino, hasta la fecha, ha sido el más castigado por su impacto (Hupkau y Victoria, 2020; Farré et al., 2020). Esta circunstancia podría dificultar considerablemente la permanencia en el mercado de trabajo de las mujeres que hayan interrumpido o reducido su participación laboral durante el confinamiento para hacer frente a responsabilidades familiares.

Analizar las dimensiones mencionadas resulta relevante en el contexto identificado por investigaciones paralelas sobre el impacto de la pandemia en la desigualdad de género en España. Se ha constatado que, si bien se dio un ligero aumento de la participación doméstica de los hombres durante el confinamiento, la carga de trabajo en el hogar continuó recayendo fundamentalmente sobre las mujeres, tal y como venía sucediendo con anterioridad (Farré et al., 2020). Estos hallazgos hacen pertinente, no obstante, indagar sobre posibles diferencias entre distintos grupos sociales y su alcance, así como sobre su relación con la situación concreta de las familias durante el confinamiento, especialmente en términos de condiciones laborales. En este sentido, las investigaciones previas sobre desigualdades de género en los usos del tiempo han prestado tradicionalmente más atención al efecto de la dedicación horaria al trabajo remunerado que a los de aspectos más cualitativos como el acceso al teletrabajo y/o la flexibilidad horaria. En los últimos años, algunas contribuciones al respecto sobre otros países han sugerido que este tipo de medidas facilitarían la implicación masculina en tareas del hogar (Giovanis, 2018), aunque también hay investigaciones previas que sugieren lo contrario (véase Noonan, 2013) y otras realizadas en el contexto de la pandemia que encuentran tendencias opuestas para el trabajo doméstico y los cuidados (Lyttelton et al., 2020). El presente estudio puede representar una aportación adicional a esta literatura, además de contribuir al conocimiento existente sobre la influencia de la pandemia en distintos tipos de desigualdad en el caso español.

\section{CONTEXTO, MARCO TEÓRICO Y PREGUNTAS DE INVESTIGACIÓN}

Las estrictas condiciones de confinamiento establecidas en España a raíz de la expansión de la COVID-19 supusieron en muchos hogares no solo el recrudecimiento de conflictos entre las exigencias domésticas y las laborales, sino también un nuevo escenario de negociación intrafamiliar. Con todo, aunque la necesidad de redistribuir los tiempos es algo a lo que se enfrentaron las familias en su conjunto, el período de confinamiento no afectó a todas 
por igual. Como se ha señalado, existen importantes diferencias relativas a la posibilidad de que ambos progenitores pudieran o no ejercer un empleo durante la fase más estricta del estado de alarma. En algunas familias se dieron condiciones favorables a la participación laboral tanto del padre como de la madre, y estos pudieron, además, ejercer su profesión en remoto y/o alternar sus respectivos horarios para compatibilizar ambos empleos con las necesidades domésticas. Puede afirmarse que este grupo, en el conjunto de la población española, constituye una minoría privilegiada, ya que se ha estimado que solo alrededor del $30 \%$ de los trabajadores comenzó a teletrabajar por primera vez a raíz de la crisis de la COVID-19 (Eurofound, 2020), y menos de un 5\% lo hacía regularmente con anterioridad (INE, 2020). Los empleos más susceptibles de llevarse a cabo desde casa son los llamados de cuello blanco, a tiempo completo, de carácter indefinido y asociados a un nivel educativo elevado (Palomino et al., 2020). Las cifras de flexibilidad horaria en España también venían siendo bajas en los últimos años, salvo en los puestos más altos de la jerarquía ocupacional (Brega Baytelman y González Pírez, 2020). En consecuencia, cabría esperar que las familias con condiciones más ventajosas para una conciliación corresponsable también partieran de una situación socioeconómica especialmente favorable.

En otros casos, por el contrario, la clausura de los centros de trabajo supuso un expediente de regulación de empleo para empleados que solo podían ejercer su profesión de manera presencial. A finales de mayo de 2020, tres millones de trabajadores en España se encontraban en esta situación. Otras personas - más de un millón de cotizantes a la seguridad social y alrededor de un millón y medio de trabajadores autónomos- perdieron su empleo como consecuencia de la paralización extensiva de la actividad económica (Doménech, 2020). La necesidad de atender a los hijos ante el cierre de los centros escolares y servicios de cuidado forzó a aquellos progenitores que mantuvieron su empleo sin posibilidad de alternancia con la pareja o teletrabajo a acogerse a una reducción de jornada dentro del llamado Plan MECUIDA. Esta reducción podía comprender hasta el $100 \%$ de la jornada laboral, con una reducción de ingresos equivalente ${ }^{3}$ (BOE, 2020). Dado que en España mayoritariamente son las madres quienes hacen uso de permisos parentales y reducciones de jornada (González y Jurado Guerrero, 2015), es muy probable que también hayan sido fundamentalmente mujeres quienes se hayan encontrado en esta situación.

3 Real Decreto Ley 8/2020, de 17 de marzo, de medidas urgentes extraordinarias para hacer frente al impacto económico y social de la COVID-19 (BOE, A-2020-3824. Disponible en: https://bit.ly/3mOtXls). 
Estas diferencias de partida, favorecedoras del mantenimiento de dos empleos en algunos hogares durante el confinamiento y no en otros, podrían haber sido cruciales para la posibilidad de establecer distribuciones del trabajo más o menos igualitarias. Las tres perspectivas teóricas habitualmente utilizadas para explicar la división de género del trabajo en las parejas predecirían, dadas las circunstancias mencionadas, una mayor probabilidad de reparto corresponsable de tareas en familias en las que ambos progenitores tuvieron la posibilidad de conservar su empleo y de hacer uso del teletrabajo y/o la flexibilidad horaria.

Uno de los principales marcos explicativos de la distribución intrafamiliar del trabajo es el conocido como teoría de la disponibilidad de tiempo. Según esta perspectiva, la dedicación de cada miembro de la pareja a las tareas domésticas y de cuidado se determinaría de forma racional en función de sus respectivos constreñimientos y compromisos temporales. En este sentido, se considera que el tiempo que cada uno ha de dedicar al trabajo remunerado juega un papel fundamental (Hiller, 1984; Coverman, 1985; Presser, 1994; Shelton y John, 1996). Esta perspectiva está respaldada por abundante literatura empírica, si bien la magnitud de los efectos varía entre países, entre hombres y mujeres, en función de si se considera la disponibilidad de tiempo propia o la de la pareja, y entre diferentes actividades (véanse, por ejemplo, Esping-Andersen, 2009; Aassve et al., 2014; Gracia y Esping-Andersen, 2005). Con respecto al objetivo concreto de este trabajo, la perspectiva de la disponibilidad de tiempo ofrece un fundamento sólido para esperar distribuciones de tareas más corresponsables en conjunto en familias en las que ambos progenitores mantuvieron su empleo. Desde un punto de vista estrictamente teórico, la dedicación por parte del padre y de la madre al trabajo remunerado durante el confinamiento habría reducido el tiempo disponible de cada uno para otras tareas, haciendo más necesario repartirlas entre ambos para poder abarcarlas en toda su magnitud. A nivel empírico, se ha constatado en investigaciones previas que el tiempo que la pareja dedica al empleo correlaciona, tanto para hombres como para mujeres, de forma positiva con la dedicación propia a las tareas domésticas, aunque esta adaptación parece ser menor en el caso de los hombres (Gershuny et al., 2005). En lo referente a los cuidados, la implicación paterna parece responder positivamente a la participación laboral de la pareja (Gutiérrez-Domènech, 2010; Gracia y Kalmijn, 2016), aunque el efecto no es uniforme en todos los países y difiere entre distintas dimensiones del cuidado (Hook and Wolfe, 2012; Gracia y Esping-Andersen, 2015). En el caso concreto de España, se ha observado que el empleo materno influye positivamente en el tiempo que dedican los padres de niños pequeños a cuidados físicos y de rutina, lo que resulta un factor clave para una mayor igualdad de género en el hogar (Gracia, 2014; Gracia y Esping-Andersen, 2015). 
Las madres en España tienden a dedicar más horas al cuidado que los padres, aún en parejas de doble ingreso (González et al., 2015). Con todo, el aumento habitual de la implicación paterna cuando la madre está empleada debería situar a estas parejas en una situación más favorable, de entrada, para alcanzar acuerdos corresponsables.

Esta expectativa parece especialmente relevante en un contexto de demandas domésticas muy intensas como el que caracterizó al confinamiento en las familias con hijos menores a cargo. Los progenitores tuvieron que hacer frente, al mismo tiempo, a necesidades de cuidado, de juego y de atención educativa, así como a una carga de tareas domésticas incrementada debido a la mayor presencia en el hogar de todos sus miembros. En familias biparentales en las que tanto padres como madres continuaron ejerciendo un trabajo remunerado, cabe suponer que fuera necesario compartir en gran medida las tareas para poder abarcarlas. El teletrabajo de ambos, a priori, debería haber aumentado la probabilidad de un reparto equitativo con respecto a situaciones en las que un progenitor trabajó fuera de casa y el otro dentro, ya que la presencia en el hogar facilita que se asuma una mayor carga doméstica (Giovanis, 2018). La flexibilidad horaria, por su parte, podría haber propiciado también un reparto corresponsable al favorecer la alternancia de roles entre los dos progenitores a lo largo del día. En los últimos años, se ha puesto el foco precisamente en estas variables como posibles facilitadoras de la conciliación y la corresponsabilidad. La flexibilidad laboral podría tener un efecto positivo en este sentido al incrementar la autonomía de los individuos para disponer de su tiempo y distribuirlo entre diferentes tareas atendiendo a sus necesidades horarias. El teletrabajo, por su parte, disminuiría los conflictos tradicionalmente derivados de la separación temporal y física de las esferas laboral y doméstica (véase Chung y Van der Lippe, 2020). Hay que tener en cuenta, no obstante, que otros factores más allá de los relacionados con la disponibilidad temporal inciden en la distribución intrafamiliar de tareas, pudiendo haber resultado importantes durante el confinamiento. Para empezar, es muy probable que las parejas cuyos miembros pudieron permanecer laboralmente activos presenten un sesgo de selección; es decir, características que podrían hacerlas, ya de entrada, más proclives tanto a conservar sus empleos como a alcanzar repartos de tareas más igualitarios. En concreto, podrían tener un mayor nivel educativo, ya que este, como se ha indicado, se asocia fuertemente en España con una mayor probabilidad de ejercer una profesión que permita realizar teletrabajo (Palomino et al., 2020). De ser el caso, cabría esperar que también pudieran presentar actitudes e ideales potencialmente favorecedores de divisiones corresponsables del trabajo, puesto que un nivel educativo elevado suele asociarse con valores de género menos tradicionales (González y Jurado Guerrero, 2009). 
Otro de los grandes marcos explicativos de la división del trabajo dentro de las parejas lo constituyen, precisamente, las teorías basadas en el género. Estas relacionan los arreglos acordados con el grado de internalización, por parte de los individuos, de las normas tradicionales que permean toda la estructura social respecto a los roles considerados adecuados para hombres y mujeres (Brines, 1993). Una adherencia normativa a roles tradicionales tendrá como resultado comportamientos menos igualitarios, mientras que unos valores y actitudes no tradicionales favorecerán divisiones del trabajo corresponsables e incluso marcadamente opuestas a la norma social, como que el padre asuma el rol de cuidador primario o que la madre sea la principal proveedora económica (véase Seiz et al., 2019). Las normas, ideologías y valores de género tienen un efecto propio sobre la división del trabajo, pero también actúan como mediadoras de la influencia de otras variables como la disponibilidad de tiempo. En este sentido, se ha observado que los hombres socializados en valores no tradicionales son especialmente proclives a incrementar su implicación en los cuidados ante una situación de desempleo (Abril et al., 2015). Por el contrario, la internalización de normas tradicionales puede llevar a la reproducción de roles normativos incluso en contextos en los que sería racional o necesario distribuir el trabajo de otra manera. Una ilustración de este fenómeno serían aquellos comportamientos englobados en la perspectiva conocida como «doing gender» (West y Zimmermann, 1987), que pone el foco en la reproducción de roles de género tradicionales en las actividades cotidianas como el reparto del trabajo. Algunas investigaciones han mostrado que en hogares en los que la mujer ejerce de único o principal proveedor económico los miembros de la pareja pueden desarrollar comportamientos tradicionales para compensar la desviación con respecto a la norma social - por ejemplo, las mujeres aumentan su dedicación al trabajo remunerado y los hombres la disminuyen (Greenstein, 2000; Bittman et al., 2003)—. Con respecto al teletrabajo y la flexibilidad, estudios recientes han señalado que podrían llegar a reforzar los roles de género tradicionales en contextos sociales en los que estos sean predominantes a nivel actitudinal y normativo. En estos casos, los hombres podrían utilizar dichas medidas para extender su implicación laboral más allá del horario oficial, y las mujeres para incrementar su dedicación familiar (Chung y Van der Lippe, 2020).

En el caso español, el hecho de que el teletrabajo y la flexibilidad horaria se asocien predominantemente a empleos en las posiciones superiores de la estructura ocupacional y a un nivel educativo elevado tiene implicaciones en términos de expectativas sobre su posible efecto sobre la corresponsabilidad en las parejas. En primer lugar, los individuos con un mayor nivel educativo tienden a adherirse en mayor medida a actitudes de género no tradicionales, por lo que cabría esperar que en este subgrupo las medidas de teletrabajo y 
flexibilidad contribuyan más a facilitar la corresponsabilidad que a fomentar divisiones tradicionales del trabajo. En segundo lugar, más allá de los factores relacionados con la disponibilidad de tiempo y los valores de género, las madres que hayan accedido a estas medidas de conciliación podrían tener una posición más favorable que otras a la hora de negociar con sus parejas arreglos corresponsables. Este punto de partida, a priori más ventajoso, se sustentaría en la teoría de los recursos relativos, otro de los grandes marcos explicativos de la división de género del trabajo (especialmente, del no remunerado). Esta perspectiva parte del supuesto de que las tareas domésticas, por lo general, constituyen actividades poco atractivas para los individuos, por lo que estos tratarán de negociar arreglos que reduzcan su implicación en las mismas. En caso de que en las negociaciones llevadas a cabo en el seno de las parejas se diera una colisión de preferencias, los recursos de cada miembro con respecto al otro serían determinantes para el resultado de la negociación, ya que podrían utilizarse en la misma para invocar algún tipo de amenaza (generalmente, la de la separación) (Lundberg y Pollak, 1996). En consecuencia, este marco teórico predice que las mujeres con una mejor posición económica tendrían mayor poder de negociación y, por tanto, un punto de partida más propicio que otras para conseguir establecer distribuciones igualitarias de tareas. Como se ha señalado, es probable que las mujeres que accedieron a medidas de teletrabajo y/o flexibilidad durante el confinamiento sean precisamente aquellas con una posición educativa y laboral más elevada. Este sería un factor adicional que podría haber favorecido divisiones corresponsables del trabajo en hogares con acceso a tales medidas por parte de ambos progenitores.

En resumen, partiendo de las perspectivas teóricas habitualmente utilizadas para explicar la división intrafamiliar del trabajo, encontramos motivos para suponer que las familias con dos progenitores empleados y con derecho a teletrabajo/flexibilidad horaria habrían estado, por diversas razones, mejor posicionadas para establecer estrategias corresponsables durante la emergencia sanitaria. Con el fin de verificar hasta qué punto fue el caso y arrojar luz sobre los factores potencialmente implicados, se formulan las siguientes preguntas de investigación:

- En las familias en las que ambos progenitores mantuvieron su empleo y accedieron conjuntamente al teletrabajo y/o la flexibilidad horaria durante el confinamiento, ¿̇se establecieron divisiones del trabajo más corresponsables que en familias que no gozaron de tales condiciones, tal y como podría esperarse desde la perspectiva de la disponibilidad de tiempo?

- Más allá de la influencia del acceso de ambos progenitores al teletrabajo y/o la flexibilidad horaria, ¿se observa algún efecto positivo del nivel 
educativo y la posición ocupacional de las madres sobre el establecimiento de arreglos corresponsables, tal y como cabría predecir desde la teoría de los recursos relativos o los marcos explicativos basados en los valores de género?

- ¿Cuál ha sido la importancia relativa, durante el confinamiento, de los factores relacionados con el empleo de cada progenitor, así como de la educación y el tipo de ocupación de la madre, a la hora de condicionar un reparto equitativo de tareas dentro de las parejas?

La exploración de estas cuestiones aportará indicaciones sobre si nos encontramos, como consecuencia de la emergencia sanitaria por COVID-19, ante una polarización social incipiente entre familias con condiciones favorables para la corresponsabilidad y el mantenimiento de un doble ingreso, y otras en posiciones en varios respectos más vulnerables.

\section{DATOS Y METODOLOGÍA}

El análisis, como se ha señalado en la sección introductoria, está basado en la encuesta "Conciliación y usos del tiempo en España en el contexto de la pandemia COVID-19", llevada a cabo entre el 9 de abril y el 11 de mayo de 2020 ( $\mathrm{n}=1287$ individuos). Debido tanto al método de reclutamiento utilizado (muestreo de bola de nieve en redes sociales) como a la administración del cuestionario a través de internet, esta encuesta no tiene un carácter representativo. Por el contrario, presenta un sesgo relacionado con el mayor acceso a una población con un nivel educativo relativamente elevado, conexión a internet y buen manejo de las nuevas tecnologías, así como probablemente concienciada con las necesidades de los procesos de investigación y las cuestiones referentes a la conciliación. Se da una sobrerrepresentación relativa de personas entre 35 y 44 años (65\%), con estudios superiores $(82 \%)$, empleadas (87\%), y con ocupaciones de carácter directivo, profesional, técnico, o de apoyo administrativo (códigos 1 a 4 en la Clasificación Internacional Uniforme de Ocupaciones (CIOU-08)) (93\%). La mayoría de las personas encuestadas tienen uno (38\%) o dos hijos (50\%), siendo en el $61 \%$ de los casos al menos alguno de ellos menor de seis años. En el apéndice se presenta una tabla descriptiva de las principales características de la muestra general. Estos sesgos impiden, como es natural, extraer conclusiones generales extrapolables al conjunto de la población española de familias con menores. Sin embargo, no deberían suponer un obstáculo significativo para el objetivo de detectar desigualdades asociadas al disfrute o no de medidas de teletrabajo/flexibilidad por parte de las madres y los padres. El 
motivo es que la submuestra relevante para este análisis — familias biparentales heterosexuales con los dos progenitores conviviendo en el mismo hogar- sí presenta una distribución equilibrada en este sentido. De las 908 familias que componen la submuestra utilizada en este estudio, el $46 \%(n=418)$ se caracterizó por el disfrute de las medidas de conciliación mencionadas por parte de ambos progenitores, mientras que en el $54 \%$ de los casos $(n=490)$ no se dio esta situación. Respecto a los otros dos factores señalados en la sección teórica como potencialmente relevantes a la hora de acentuar desigualdades — el nivel educativo de la madre y su tipo de ocupación-, la variabilidad es suficiente para permitir un análisis cuantitativo (el $82 \%$ de las mujeres analizadas han completado estudios superiores y el $18 \%$ no lo han hecho; el $54 \%$ desempeñan ocupaciones profesionales en el nivel 2 del CIOU, pero el resto están repartidas entre los distintos niveles jerárquicos).

El motivo para centrar el trabajo en familias con dos progenitores conviviendo en el hogar es que se busca examinar el resultado de las negociaciones cotidianas de las parejas confinadas junto a sus hijos. Se incluyen solo familias heterosexuales por dos razones: por un lado, el limitado número de familias homosexuales representado en la encuesta (diez observaciones) imposibilitaba cualquier análisis sustantivo. Por otro lado, en el foco de este artículo está la brecha de género en la división del trabajo dentro de las parejas. Se parte exclusivamente de personas encuestadas de sexo femenino $(\mathrm{n}=908)$, ya que solo estas proporcionan información sobre el tipo de ocupación y el nivel educativo maternos; variables claves en el análisis. La muestra equivalente de hombres, además, habría resultado demasiado reducida $(\mathrm{n}=216)$ para permitir un análisis en subgrupos.

El análisis, de naturaleza mayormente descriptiva, comienza con el examen de las características de los dos grupos en los que se divide la muestra de interés, con el fin de detectar probables sesgos de selección, en términos socioeconómicos, entre las familias en las que ambos progenitores se pudieron acoger a medidas de teletrabajo/flexibilidad, y aquellas en las que no fue el caso. Se incluye a las familias en el primer subgrupo si tanto el padre como la madre hicieron uso del teletrabajo o si ambos pudieron recurrir a la flexibilidad horaria. Se presta particular atención al nivel educativo de la madre, desglosado en las categorías que se presentan en la tabla 1, así como a su posición en la jerarquía ocupacional según el CIOU-08.

A continuación, para responder a la primera pregunta de investigación formulada en la sección teórica — es decir, para averiguar si las familias en las que ambos progenitores accedieron a teletrabajo/flexibilidad establecieron divisiones de tareas más corresponsables durante el confinamiento-, se procede de entrada a explorar los usos absolutos del tiempo en cada subgrupo familiar. En particular, se consideran las 
horas medias dedicadas por las mujeres y sus parejas, respectivamente, al trabajo remunerado, el trabajo doméstico rutinario (comida, limpieza, compra), los cuidados físicos y de rutina (alimentación, higiene, vestido, vigilancia), el juego con los niños y la atención educativa. Con el fin de capturar de forma más precisa cómo fue la distribución de los tiempos en términos relativos, se calcula también el porcentaje que asumieron, en promedio, las madres y los padres en cada subgrupo con respecto a cada tipo de trabajo. La finalidad de esta medida es ver hasta qué punto las tareas se dividieron o no de manera igualitaria - entendiendo como tal una distribución en la que ningún progenitor realizó menos del $40 \%$ de la tarea en cuestión ni más del $60 \%$ - en cada categoría de familias. Por último, se cuantifica qué porcentaje de parejas en cada una de ellas alcanzó distribuciones del trabajo igualitarias (según la definición mencionada), tradicionales (la madre realizó menos del $40 \%$ de las horas en el caso del trabajo remunerado y más del $60 \%$ en el caso del trabajo no remunerado), y no normativas (la mujer realizó más del $60 \%$ de las horas en el caso del trabajo remunerado y menos del $40 \%$ en el caso del trabajo no remunerado). Los umbrales de $40 \%$ y $60 \%$, utilizados para distinguir los repartos igualitarios de los tradicionales y no normativos, respectivamente, responden a convenciones habituales en la literatura sobre la división del trabajo en el seno de las parejas (véanse por ejemplo, Nock, 2001; Esping-Andersen et al., 2013; Domínguez-Folgueras et al., 2018; González et al., 2018). Para complementar la información descriptiva sobre la primera pregunta de investigación y corroborar si el acceso conjunto a medidas de teletrabajo/flexibilidad hizo más probable un reparto de tareas corresponsable entre los progenitores, se recurre a modelos de regresión logística. Estos permiten también responder a la segunda pregunta de investigación y examinar un posible efecto adicional del nivel educativo y la posición ocupacional de la madre. Se ejecuta un modelo similar para cada una de las tareas de interés (trabajo remunerado, tareas domésticas, cuidados físicos y de rutina, juego, y atención educativa). La variable dependiente es, en todos los casos, una variable dicotómica que recoge si se estableció una división corresponsable del trabajo (entendida, como se ha explicado arriba, como aquella en la que ninguno de los miembros de la pareja realizó más del $60 \%$ de la tarea en cuestión ni menos del $40 \%$ ). Como variables independientes se incorporan otras variables dicotómicas que miden si ambos progenitores hicieron uso del teletrabajo y/o la flexibilidad; si la madre tenía estudios universitarios, y si tenía una ocupación clasificada en los niveles 1 o 2 del CIOU-08 (directivas o profesionales). Como controles se incluyen variables dicotómicas que reflejan si había más de un niño en la familia y la presencia de menores de seis años, respec- 
tivamente. Por último, se analiza asimismo la importancia relativa de las dos características maternas mencionadas frente a los factores relacionados con el empleo de cada progenitor mediante modelos de regresión que incluyen, para cada uno por separado, medidas dicotómicas de su situación laboral (empleado vs. no empleado), el acceso al teletrabajo y el acceso a la flexibilidad horaria. Estos modelos se realizan también para cada actividad de trabajo remunerado y no remunerado, siendo la variable dependiente, de nuevo, el establecimiento de una división corresponsable del trabajo durante el período de confinamiento.

\section{RESULTADOS}

En la tabla 1 se presentan las características generales de la muestra de mujeres analizada, que se ha dividido en los dos subgrupos claves para esta investigación: uno en el que ambos progenitores hicieron uso de medidas de teletrabajo/flexibilidad durante el confinamiento, y otro en el que no fue el caso. Como se puede observar, los dos subgrupos son bastante similares en cuanto a distribución de edades, si bien el segundo está compuesto, en conjunto, por individuos algo más jóvenes. En los dos casos predominan las mujeres con estudios universitarios, aunque el nivel educativo es más elevado en el primer subgrupo (el $42 \%$ de las madres tienen estudios de máster y doctorado, frente al $29 \%$ del segundo). El segundo subgrupo cuenta, además, con una proporción visiblemente mayor de mujeres con estudios de educación secundaria o inferiores (13,5\%, frente al 3\% del primero). Respecto a la situación laboral, en el segundo subgrupo hay un $27 \%$ de mujeres que declaran no estar empleadas. También hay diferencias en cuanto a la posición de la mujer en la estructura ocupacional, ya que en el primer subgrupo se da una proporción mayor de encuestadas en ocupaciones directivas $(8,6 \%$ frente a $5,7 \%)$ y de profesionales técnicas e intelectuales $(63,4 \%$ frente a $46,7 \%)$. Como contraste, en el segundo subgrupo se observa una proporción relativamente mayor de personal de apoyo administrativo $(18,4 \%$ frente a $9,6 \%)$ y de trabajadoras de los sectores comercial y de servicios $(7,4 \%$ frente al 3,8\%). En resumen, existe una diferencia de partida entre las dos categorías de familia estudiadas basada en el nivel educativo y la posición en la jerarquía ocupacional. Los dos son más elevados en aquellas familias en las que ambos progenitores gozaron de acceso a teletrabajo/flexibilidad durante el confinamiento, confirmando el sesgo de selección postulado en la sección teórica.

Un primer análisis del promedio diario de horas dedicadas al trabajo remunerado, las tareas domésticas, los cuidados, el juego con los hijos, y la 
educación de estos revela, en primer lugar, diferencias de género en el empleo del tiempo dentro de las familias durante el período de confinamiento. Estas diferencias son evidentes tanto en familias en las que ambos progenitores tuvieron acceso a medidas de teletrabajo/flexibilidad, como en aquellas en las que no se dio esta circunstancia, aunque resultan más marcadas en el último grupo. Como se puede apreciar en el gráfico 1, los padres dedicaron, en cualquiera de los casos, más tiempo al trabajo remunerado que las madres, si bien la distancia fue mayor (casi dos horas más) en el segundo grupo que en el primero (la diferencia entre el padre y la madre no llega en este caso a una hora). En el grupo en el que ambos progenitores pudieron conservar su empleo y hacer uso conjunto del teletrabajo y/o la flexibilidad horaria, las madres dedicaron una media de algo más de seis horas a su profesión, y los padres algo más de siete. En el grupo en el que los progenitores no pudieron hacer uso simultáneo de tales medidas de conciliación, las madres realizaron casi cuatro horas diarias de trabajo remunerado y los padres unas cinco y media en promedio. Esta menor cantidad de horas, con respecto al grupo anterior, se debe probablemente al hecho de que el segundo grupo recoge una cierta proporción de personas que no pudieron trabajar $(27,1 \%$ en el caso de las mujeres y $16,5 \%$ en el caso de los hombres), tal y como pone de manifiesto la tabla 1.

Gráfico 1. Dedicación media diaria a distintos tipos de trabajo en familias biparentales en función del acceso o no de ambos progenitores a medidas de teletrabajolflexibilidad

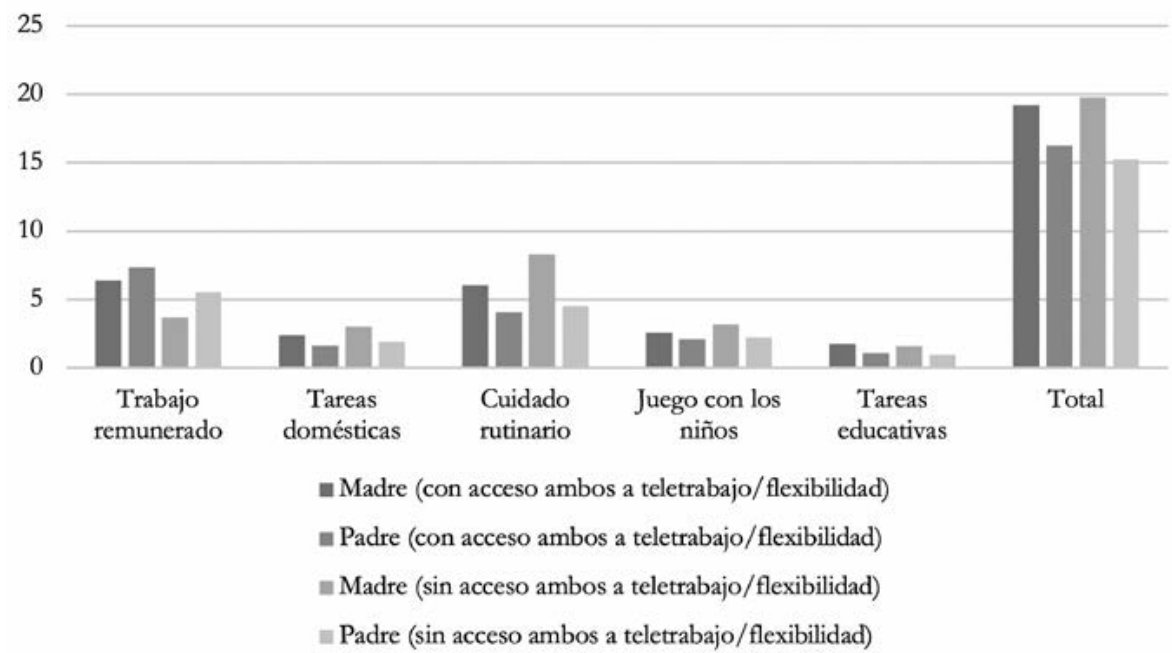

Fuente: elaboración propia. 
Tabla 1. Características demográficas y socioeconómicas de las mujeres en los subgrupos de análisis y sus parejas

\begin{tabular}{|c|c|c|}
\hline & $\begin{array}{l}\text { Parejas en las que } \\
\text { ambos progenitores } \\
\text { hicieron uso } \\
\text { de teletrabajo/ } \\
\text { flexibilidad }\end{array}$ & $\begin{array}{l}\text { Parejas en otras } \\
\text { situaciones }\end{array}$ \\
\hline $\begin{array}{l}\text { Edad de la madre } \\
25-34 \text { años } \\
35-44 \text { años } \\
>45 \text { años } \\
\text { Valores perdidos }\end{array}$ & $\begin{array}{c}8,8 \% \\
69,9 \% \\
21,3 \% \\
-\end{array}$ & $\begin{array}{l}12,4 \% \\
69,2 \% \\
18,4 \% \\
- \\
\end{array}$ \\
\hline $\begin{array}{l}\text { Nivel de estudios de la madre } \\
\text { Doctorado } \\
\text { Máster } \\
\text { Licenciatura, diplomatura o grado } \\
\text { Formación profesional de cualquier ciclo } \\
\text { Enseñanza general secundaria de segundo ciclo } \\
\text { Enseñanza general secundaria de primer ciclo } \\
\text { Estudios primarios o sin estudios } \\
\text { Valores perdidos }\end{array}$ & $\begin{array}{c}12,0 \% \\
30,4 \% \\
48,1 \% \\
6,2 \% \\
2,6 \% \\
0,5 \% \\
- \\
0,2 \%\end{array}$ & $\begin{array}{c}5,1 \% \\
24,3 \% \\
45,9 \% \\
11,2 \% \\
8,6 \% \\
4,1 \% \\
0,8 \% \\
-\end{array}$ \\
\hline $\begin{array}{l}\text { Situación laboral de la madre } \\
\text { Empleada/o (por cuenta ajena o propia) } \\
\text { Desempleada } \\
\text { No empleada, otra situación } \\
\text { Ama de casa } \\
\text { Valores perdidos }\end{array}$ & $\begin{array}{c}98,5 \% \\
0,5 \% * \\
1,0 \% * \\
- \\
-\end{array}$ & $\begin{array}{c}72,9 \% \\
10,6 \% \\
9,2 \% \\
7,3 \% \\
-\end{array}$ \\
\hline $\begin{array}{l}\text { Profesión (CIOU-08) de la madre } \\
\text { Directoras y gerentes } \\
\text { Profesionales técnicos e intelectuales } \\
\text { Técnicas y profesionales de nivel medio } \\
\text { Personal de apoyo administrativo } \\
\text { Trabajadoras de los servicios y vendedoras } \\
\text { de comercios y mercados } \\
\text { Agricultoras y trabajadoras calificadas } \\
\text { agropecuarias, forestales y pesqueras } \\
\text { Oficiales, operarias y artesanas } \\
\text { de artes mecánicas y de otros oficios } \\
\text { Operadoras de instalaciones de máquinas } \\
\text { y ensambladoras } \\
\text { Ocupaciones elementales } \\
\text { Valores perdidos }\end{array}$ & $\begin{array}{c}8,6 \% \\
63,4 \% \\
10,1 \% \\
9,6 \% \\
3,8 \% \\
- \\
0,2 \% \\
\\
- \\
0,2 \% \\
4,1 \%\end{array}$ & $\begin{array}{l}5,7 \% \\
46,7 \% \\
9,6 \% \\
18,4 \% \\
7,4 \% \\
\\
- \\
0,4 \% \\
\\
0,4 \% \\
1,8 \% \\
9,6 \%\end{array}$ \\
\hline
\end{tabular}


Tabla 1. Características demográficas y socioeconómicas de las mujeres en los subgrupos de análisis y sus parejas (continuación)

\begin{tabular}{|c|c|c|}
\hline & $\begin{array}{l}\text { Parejas en las que } \\
\text { ambos progenitores } \\
\text { hicieron uso } \\
\text { de teletrabajo/ } \\
\text { flexibilidad }\end{array}$ & $\begin{array}{l}\text { Parejas en otras } \\
\text { situaciones }\end{array}$ \\
\hline \multicolumn{3}{|l|}{ Situación laboral de la pareja } \\
\hline Pareja empleada (por cuenta ajena o propia) & $98,1 \%$ & $83,5 \%$ \\
\hline Pareja desempleada & $0,5 \% *$ & $8,6 \%$ \\
\hline Pareja no empleada, otra situación & $1,4 \% *$ & $6,9 \%$ \\
\hline Pareja ama de casa & - & $1,0 \%$ \\
\hline Valores perdidos & - & - \\
\hline \multicolumn{3}{|l|}{ Número de hijos presentes en el hogar } \\
\hline Uno & $36,4 \%$ & $38,4 \%$ \\
\hline Dos & $52,4 \%$ & $49,8 \%$ \\
\hline Tres & $10,0 \%$ & $10,6 \%$ \\
\hline Cuatro o más & $1,2 \%$ & $1,2 \%$ \\
\hline \multicolumn{3}{|l|}{ Presencia de hijos menores de 3 años } \\
\hline Sí & $32,8 \%$ & $37,1 \%$ \\
\hline No & $67,2 \%$ & $62,7 \%$ \\
\hline Valores perdidos & - & $0,2 \%$ \\
\hline \multicolumn{3}{|l|}{ Presencia de hijos menores de 6 años } \\
\hline Sí & $63,9 \%$ & $63,9 \%$ \\
\hline No & $36,1 \%$ & $35,9 \%$ \\
\hline Valores perdidos & - & $0,2 \%$ \\
\hline
\end{tabular}

*Estas cifras corresponden a personas que realizan trabajo remunerado (han indicado que realizan varias horas al día) a pesar de declarar no encontrarse empleadas (tampoco son autónomas). Como probablemente se trate de personas que están trabajando sin contrato, se incluyen en el análisis y en este subgrupo de familias.

Fuente: elaboración propia.

En lo que respecta al trabajo no remunerado, la dedicación de las madres fue mayor en los dos grupos, pero las diferencias de género son más visibles, de nuevo, en el segundo. En el grupo de familias en las que ambos progenitores teletrabajaron o hicieron uso de la flexibilidad laboral, las mujeres realizaron, en promedio, algo más de dos horas diarias de tareas domésticas, siendo la diferencia con respecto a sus parejas de algo menos de una hora. En el grupo de familias en las que alguno de los progenitores (o los dos) no pudo recurrir a las medidas mencionadas, las madres realizaron tres horas de trabajo doméstico en promedio y los hombres algo menos de dos. Todas las diferencias son aún 
más acentuadas con respecto al cuidado. En la muestra de familias con acceso a medidas de conciliación tanto para el padre como para la madre, las mujeres dedicaron algo más de seis horas diarias a los cuidados físicos y de rutina, dos horas más que sus parejas. En el subgrupo sin acceso conjunto a medidas de conciliación, la brecha fue considerablemente mayor: ellas invirtieron más de ocho horas diarias en cuidar, y su implicación prácticamente duplicó la de sus parejas. La distancia se acorta en lo referente al juego con los hijos, si bien fueron de nuevo las madres — especialmente en familias sin acceso de ambos progenitores al teletrabajo/la flexibilidad horaria- quienes mostraron una mayor dedicación (algo más de tres horas diarias; una más que sus parejas, en el caso de este grupo en concreto). En las familias en las que ambos padres pudieron teletrabajar o recurrir a horarios flexibles, la diferencia fue de media hora, dedicando las madres algo más de dos horas y media diarias a esta tarea. Por último, la atención educativa también recayó fundamentalmente sobre las madres, y entre ellas fueron las del primer subgrupo las que más tiempo destinaron a esta labor (una hora y tres cuartos al día; seguidas de cerca por las madres del segundo subgrupo. Los hombres, en los dos subgrupos, dedicaron en promedio alrededor de una hora).

En resumen, analizando la dedicación horaria absoluta a distintos tipos de trabajo durante el confinamiento, se observa que los padres destinaron más tiempo que las madres al trabajo remunerado, incluso en parejas en los que ambos tuvieron acceso al teletrabajo y/o la flexibilidad horaria, mientras que las mujeres realizaron más horas de trabajo no remunerado. Estas diferencias por género, no obstante, fueron menores en estas últimas familias que en aquellas en las que los progenitores no pudieron hacer uso conjunto de tales medidas. Es importante destacar que las madres en este segundo subgrupo fueron quienes asumieron una mayor carga de trabajo total, realizando, en promedio, casi veinte horas diarias de trabajo remunerado y no remunerado. La cifra media correspondiente a las madres del primer subgrupo fue solo ligeramente menor - en total, asumieron una carga de trabajo equivalente a poco más de diecinueve horas- Estas cifras contrastan visiblemente con las correspondientes al total de trabajo diario realizado por los hombres. La carga de trabajo asumida por los padres fue de tres horas menos que la correspondiente a las madres en caso del primer subgrupo, y de casi cinco horas menos en el caso del segundo.

La elaboración de medidas relativas que cuantifican exactamente la magnitud de la brecha de género en cada tipo familia y por tipo de trabajo nos permite, con todo, matizar los resultados anteriores. En concreto, se analiza si las parejas en las que ambos miembros hicieron uso de teletrabajo/flexibilidad horaria se aproximaron o no, en términos generales, a una división corresponsable del trabajo, y en qué medida se diferencian del segundo grupo en este sentido. Los gráficos 2 y 3 muestran el porcentaje del trabajo diario 
Gráfico 2. Proporción media (\%) de la carga de trabajo diario asumida por madres y padres en familias en las que ambos progenitores hicieron uso de teletrabajolflexibilidad horaria durante el confinamiento

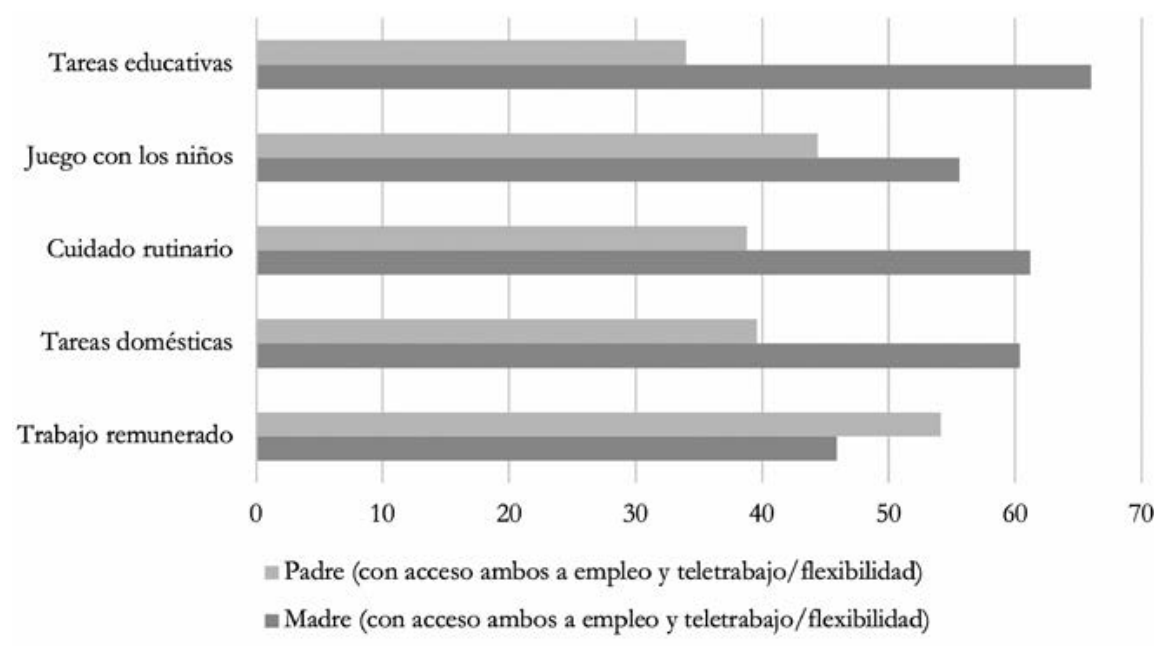

Fuente: elaboración propia.

Gráfico 3. Proporción media (\%) de la carga de trabajo diario asumida por madres y padres en familias en las que los progenitores no hicieron uso conjunto de teletrabajolflexibilidad horaria durante el confinamiento

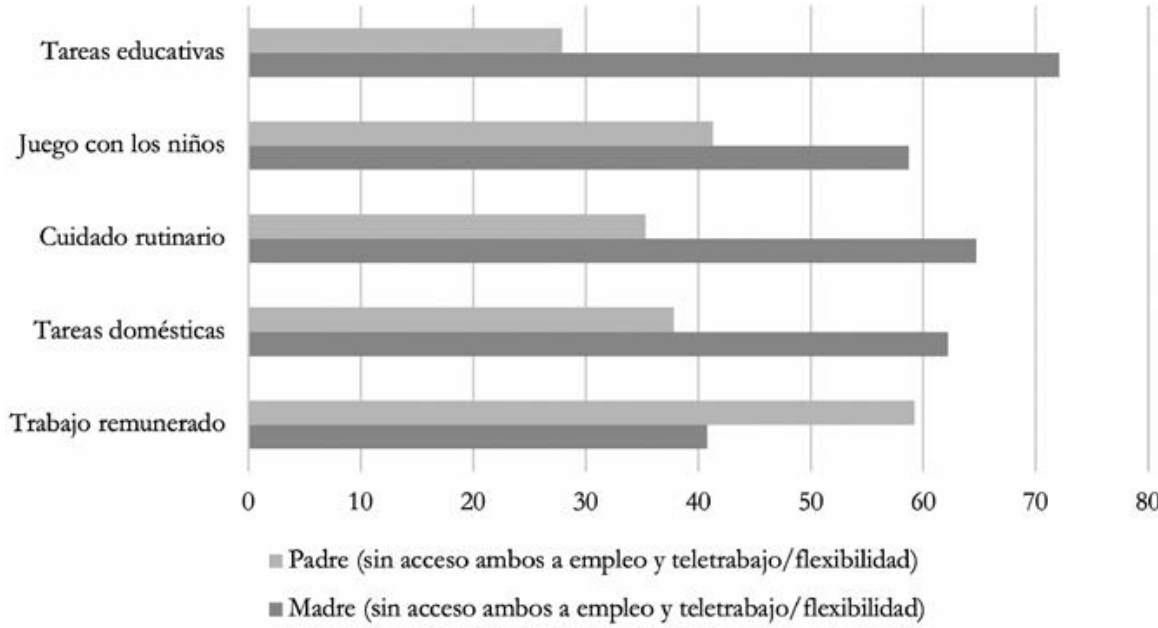

Fuente: elaboración propia. 
asumido, en promedio, por madres y padres en los dos tipos de familias biparentales analizadas. Los resultados obtenidos revelan diferencias relativas a la igualdad de género en la división del trabajo entre los dos subgrupos. Aunque las madres, como se ha expuesto, asumieron una mayor carga de trabajo en términos absolutos, en las familias en las que ambos progenitores mantuvieron su empleo y pudieron hacer uso de teletrabajo y/o flexibilidad horaria, respectivamente, se alcanzaron distribuciones que pueden clasificarse como igualitarias en el trabajo remunerado y en el juego con los niños. Las cifras correspondientes al reparto relativo de las tareas domésticas y de cuidado rutinario en este grupo, aunque no corresponden estrictamente a una distribución igualitaria, se aproximan mucho - las madres asumieron poco más del $60 \%$ y los padres poco menos del $40 \%$ - Solo las tareas educativas recayeron de forma más evidente sobre las madres, que realizaron, en promedio, el $66 \%$ de las mismas. En el grupo de familias sin acceso a teletrabajo/flexibilidad por parte de los dos progenitores, las tendencias observadas son en gran medida semejantes a las descritas, aunque con mayor participación femenina relativa en la mayoría de las tareas. Solo el trabajo remunerado ${ }^{4}$ y el juego se distribuyeron de tal forma que entran dentro de los umbrales de distribución corresponsable. El resto de las tareas fueron asumidas en más de un $60 \%$ por las madres. Entre ellas destaca, una vez más, la atención educativa (en promedio, las madres de este grupo proporcionaron el $72 \%$ de la misma).

Otra medida del grado de desigualdad de género encontrado en los dos grupos de familias estudiadas la proporciona la cuantificación de los porcentajes de parejas que establecieron, en cada uno de ellos, distribuciones tradicionales, igualitarias o no normativas de cada actividad, de acuerdo con los umbrales descritos en la sección de metodología. En el gráfico 4 se compara la prevalencia de cada una de estas distribuciones en los dos subgrupos analizados con respecto a cada tipo de tarea. Esta representación nos permite hacernos una idea de la frecuencia de cada tipo de división del trabajo en función de la situación laboral de las parejas. Se puede constatar, en primer lugar, que las divisiones corresponsables del trabajo - para cualquiera de las tareas examinadas - fueron más frecuentes durante el confinamiento entre aquellas parejas en las que ambos progenitores desempeñaron un empleo con acceso a medidas de teletrabajo/flexibilidad.

Las diferencias son evidentes en lo que respecta al trabajo remunerado. Entre las familias en las que ambos progenitores mantuvieron su empleo

4 Para hacer el cálculo de la distribución relativa de las horas de trabajo remunerado dentro de las parejas solo se han incluido aquellos casos en los que al menos un progenitor ha trabajado, lo que en el caso del segundo subgrupo equivale a 436 familias. 
y pudieron utilizar medidas de conciliación, este se distribuyó de forma igualitaria en el $73 \%$ de los casos, y de forma tradicional solo en el $7 \%$ (las distribuciones no normativas, por su parte, ascendieron al $20 \%$ ). Respecto al resto de las familias, solo el $25 \%$ alcanzaron divisiones igualitarias de las horas dedicadas al empleo, y el $26 \%$ las dividieron de forma tradicional. Llama la atención en este segundo subgrupo, por otra parte, la cantidad de parejas (49\%) que establecieron distribuciones no normativas del trabajo remunerado durante el confinamiento. Esta cifra debe interpretarse con mucha cautela, ya que podrían haber influido en ella dos factores: en esta categoría de familias, el $17 \%$ de los padres no estuvo trabajando, y se han eliminado también del cálculo de distribuciones relativas del trabajo remunerado aquellas familias en las que ningún progenitor trabajó. Esto significa que podría haber una proporción no despreciable de familias en este grupo en las que la madre fue la única proveedora económica. El cálculo de este porcentaje revela que es el caso —el 21,4\% de las parejas de este subgrupo se encontró en esta situación, lo que en parte explica la cifra relativamente elevada de distribuciones no normativas-. Con todo, persiste casi un $28 \%$ de parejas que establecieron este tipo de acuerdos aun cuando ambos progenitores declaraban haber trabajado.

En el grupo de familias con acceso a teletrabajo/flexibilidad para el padre y la madre se observa, asimismo, una mayor proporción de distribuciones corresponsables de tareas domésticas que en el segundo (50\% frente al 43\%). También se aprecia una menor proporción de distribuciones tradicionales (44\% frente al $48 \%$ ). Las distribuciones no normativas de las tareas domésticas fueron algo más frecuentes en el segundo grupo (casi el $9 \%$ del total, frente al casi $6 \%$ del primer grupo), pero es probable que se deba a la circunstancia arriba mencionada - habrá parejas en las que la madre haya asumido la totalidad del trabajo remunerado por encontrarse el padre desempleado, y cabe imaginar que en esta circunstancia haya sido más fácil que él asuma una cantidad significativa de tareas domésticas debido a una mayor disponibilidad de tiempo-.

En lo que se refiere al cuidado de rutina, se aprecian diferencias más acentuadas entre los subgrupos. En la categoría de familias con acceso a medidas de conciliación para ambos progenitores, la mayoría de las divisiones del cuidado (55\%) fueron igualitarias, mientras que solo el $41 \%$ de las parejas optaron por distribuciones del tiempo tradicionales. En el segundo subgrupo, por el contrario, la tendencia fue la inversa: los arreglos tradicionales fueron predominantes (54\%), y solo el $40 \%$ de las parejas establecieron acuerdos corresponsables. Las divisiones del cuidado no normativas — en las que el padre asumió más del $60 \%$ del mismo- fueron minoritarias en ambos grupos. Se observa una incidencia ligeramente más elevada en el segundo, posiblemente por los motivos de disponibilidad de tiempo ya señalados. En lo que respecta al juego con los niños, la tendencia mayoritaria en el primer grupo también 
fue la distribución corresponsable (56\% de las parejas), mientras que en el segundo grupo las proporciones correspondientes a los arreglos tradicionales e igualitarios fueron muy similares ( $43 \%$ frente a $42 \%$ ). Cabe destacar que en ambos grupos los arreglos entendidos como no normativos - en el sentido de que el padre asumió más del $60 \%$ de las horas realizadas-fueron visiblemente más frecuentes en el juego que en el cuidado. Este hallazgo es coherente con investigaciones previas que detectan una menor brecha de género en actividades lúdicas que en aquellas de carácter rutinario (Craig y Mullan, 2011).

La implicación en las tareas educativas de los hijos merece mención aparte, ya que en este ámbito predominaron las distribuciones tradicionales - con la madre haciéndose cargo de más del $60 \%$ de las tareas-en los dos grupos de familias estudiados (en el $51 \%$ y el $59 \%$ de los casos, respectivamente, se dio este patrón durante el confinamiento). Los arreglos igualitarios, no obstante, fueron más comunes también en el primer grupo que en el segundo (38\% frente a $30 \%$ ). En ambos casos, las familias en las que el padre asumió más del $60 \%$ de las tareas educativas constituyeron alrededor del $11 \%$ del total.

En resumen, las familias en las que ambos progenitores mantuvieron el empleo con acceso a medidas de teletrabajo/flexibilidad horaria durante el confinamiento alcanzaron en mayor medida arreglos corresponsables en la división del trabajo remunerado y no remunerado, tal y como cabía esperar desde la perspectiva de la disponibilidad de tiempo. Con todo, hay que preguntarse si esta asociación se explica mayormente por el disfrute conjunto de las medidas de conciliación mencionadas, o si responde a un sesgo de selección relacionado con un nivel educativo y una posición profesional más elevada por parte de las madres que pudiera conferirles un mayor poder de negociación y mayor predisposición a negociar arreglos corresponsables. Otra posibilidad es que estas dos últimas variables muestren un efecto independiente y adicional al de las relacionadas con la disponibilidad de tiempo sobre la probabilidad de establecimiento de estrategias corresponsables durante el confinamiento. Los modelos de regresión presentados en la tabla 2 incluyen simultáneamente todas las variables mencionadas, así como controles correspondientes al número y la edad de los hijos. Como se puede observar, el uso conjunto del teletrabajo/ la flexibilidad horaria por parte de ambos progenitores muestra una asociación positiva y significativa con la división corresponsable de la práctica totalidad de las actividades consideradas ${ }^{5}$. Esta relación es especialmente fuerte en lo que respecta a las horas de trabajo remunerado. El nivel educativo de la madre no muestra en los modelos ninguna asociación significativa con una distribución

5 En el caso de las tareas domésticas, la asociación es positiva, pero no es significativa con un nivel de confianza del 95\%. Sí lo sería con un nivel de confianza del 90\%. 
Gráfico 4. Proporción (\%) de parejas que adoptaron distribuciones tradicionales, igualitarias y no normativas del trabajo remunerado y no remunerado en las dos submuestras de interés

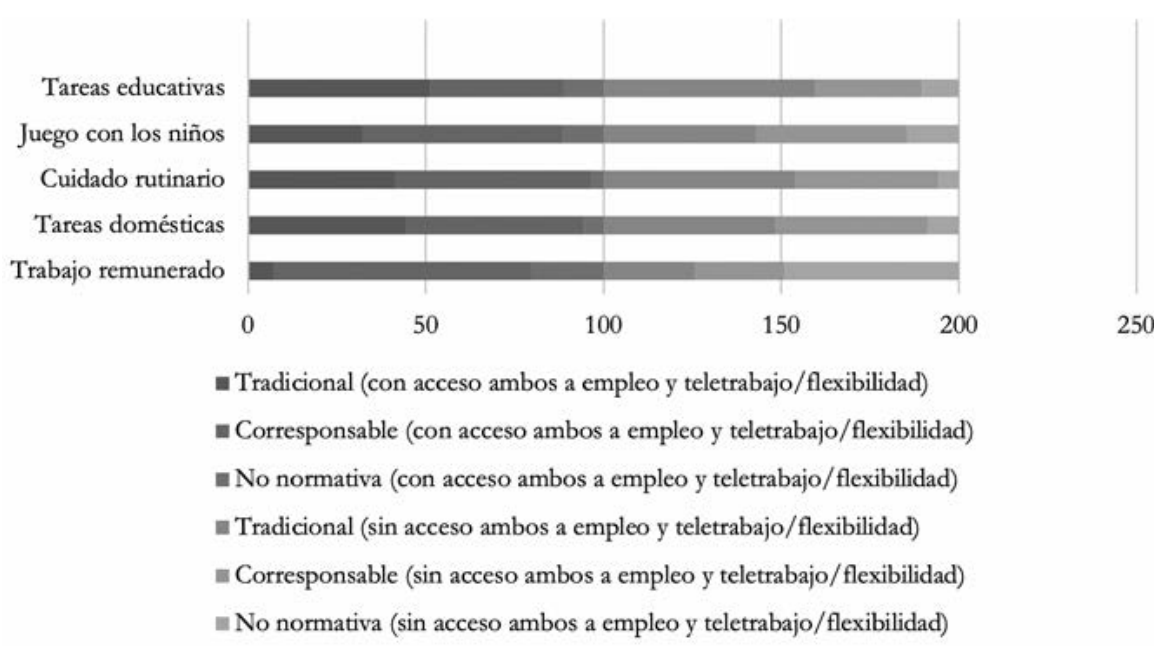

Fuente: elaboración propia.

más igualitaria del trabajo durante el período de emergencia sanitaria. Tampoco lo hace el hecho de que esta tuviera una posición elevada en la jerarquía ocupacional, salvo en el caso de las horas de trabajo remunerado, en el que se intuye un posible efecto (la asociación es positiva y significativa con un nivel de confianza del 90\%). Estos resultados no varían si se introducen las variables de nivel educativo y tipo de ocupación por sí solas, sin tener en cuenta el uso del teletrabajo y la flexibilidad horaria, lo que indica que las últimas no enmascaran un posible efecto de las primeras ${ }^{6}$.

En los modelos presentados en la tabla 3 se busca desentrañar exactamente qué elementos del uso conjunto del teletrabajo/flexibilidad por parte de ambos progenitores se asocia con la mayor probabilidad de distribución corresponsable del trabajo. Como se puede apreciar, las divisiones igualitarias del trabajo remunerado muestran una asociación positiva y fuerte, como cabía suponer, con el empleo del padre y la madre, así como con el teletrabajo de ambos. La flexibilidad horaria por parte del padre también se asoció con una mayor frecuencia de divisiones igualitarias de las horas de trabajo remunerado, pero no así la de la madre — en este caso, el efecto es negativo-. Hay que destacar que en este

6 Estos resultados no se muestran por razones de espacio, pero están disponibles bajo petición. 
Tabla 2. Regresiones logísticas de la probabilidad de haber alcanzado divisiones corresponsables del trabajo remunerado y no remunerado durante el confinamiento (coeficientes expresados como odds ratio, errores estándar entre paréntesis)

\begin{tabular}{|l|c|c|c|c|c|}
\hline & $\begin{array}{c}\text { División } \\
\text { igualitaria } \\
\text { del trabajo } \\
\text { remunerado }\end{array}$ & $\begin{array}{c}\text { División } \\
\text { igualitaria } \\
\text { de las tareas } \\
\text { domésticas }\end{array}$ & $\begin{array}{c}\text { División } \\
\text { igualitaria } \\
\text { del cuidado } \\
\text { físico y de } \\
\text { rutina }\end{array}$ & $\begin{array}{c}\text { División } \\
\text { igualitaria } \\
\text { de las } \\
\text { actividades de } \\
\text { juego }\end{array}$ & $\begin{array}{c}\text { División } \\
\text { igualitaria } \\
\text { de las } \\
\text { actividades } \\
\text { educativas }\end{array}$ \\
\hline $\begin{array}{l}\text { Uso del teletrabajo } \\
\text { / la flexibilidad por } \\
\text { parte de ambos } \\
\text { progenitores }\end{array}$ & $\begin{array}{c}7.11^{* * *} \\
(1.16)\end{array}$ & $\begin{array}{c}1.30\left(^{*}\right) \\
(.19)\end{array}$ & $\begin{array}{c}1.79^{* * *} \\
(.26)\end{array}$ & $\begin{array}{c}1.82^{* * *} \\
(.27)\end{array}$ & $\begin{array}{c}1.44^{*} \\
(.23)\end{array}$ \\
\hline $\begin{array}{l}\text { Madre con estudios } \\
\text { universitarios }\end{array}$ & $.93(.26)$ & $.98(.23)$ & $1.01(.24)$ & $.83(.19)$ & $.89(.23)$ \\
\hline $\begin{array}{l}\text { Madre en } \\
\text { ocupación de } \\
\text { carácter directivo } \\
\text { o profesional } \\
\text { (CIOU-08 1 o 2) }\end{array}$ & $1.45\left(^{*}\right)$ & $.96(.17)$ & $1.06(.19)$ & $.98(.18)$ & $1.09(.22)$ \\
\hline $\begin{array}{l}\text { Más de un menor } \\
\text { en el hogar }\end{array}$ & $1.14(.19)$ & $.99(.14)$ & $1.04(.15)$ & $1.00(.15)$ & $.70^{*}(.12)$ \\
\hline $\begin{array}{l}\text { Presencia de } \\
\text { menores de 6 años } \\
\text { en el hogar }\end{array}$ & $.68^{*}(.11)$ & $1.07(.16)$ & $.86(.13)$ & $.65^{* * *}(.10)$ & $1.23(.20)$ \\
\hline Constante & $.36^{* * *}(.09)$ & $.77(.17)$ & $.71(.17)$ & $1.12(.26)$ & $.49^{* *}(.12)$ \\
\hline Pseudo R2 & .17 & .003 & .02 & .02 & .01 \\
\hline
\end{tabular}

Fuente: elaboración propia.

modelo, al controlar por diferentes variables de disponibilidad de tiempo por separado, sí emerge una relación positiva entre un estatus ocupacional elevado por parte de la madre y la división igualitaria de las horas de trabajo en el seno de la pareja. Las divisiones igualitarias del trabajo doméstico solo se asocian de forma positiva y significativa con el hecho de que la madre estuviera empleada durante el confinamiento, así como con la flexibilidad horaria por parte de padre. La flexibilidad horaria materna, por el contrario, parece guardar relación con una mayor desigualdad en el trabajo doméstico.

Los factores que incidieron, a su vez, en la probabilidad de una distribución igualitaria del cuidado de rutina durante el confinamiento fueron el empleo y el teletrabajo maternos, así como la flexibilidad horaria por parte del padre. Como en el caso del trabajo doméstico, la flexibilidad horaria por parte de la madre se asoció negativamente con el reparto corresponsable de las 
Tabla 3. Regresiones logísticas de la probabilidad de haber alcanzado divisiones corresponsables del trabajo remunerado y no remunerado durante el confinamiento. Variables independientes de empleo desglosadas para cada progenitor

\begin{tabular}{|c|c|c|c|c|c|}
\hline & $\begin{array}{c}\text { División } \\
\text { igualitaria } \\
\text { del trabajo } \\
\text { remunerado }\end{array}$ & $\begin{array}{l}\text { División } \\
\text { igualitaria } \\
\text { de las tareas } \\
\text { domésticas }\end{array}$ & $\begin{array}{l}\text { División } \\
\text { igualitaria } \\
\text { del cuidado } \\
\text { físico y de } \\
\text { rutina }\end{array}$ & $\begin{array}{l}\text { División } \\
\text { igualitaria } \\
\text { de las } \\
\text { actividades } \\
\text { de juego }\end{array}$ & $\begin{array}{l}\text { División } \\
\text { igualitaria } \\
\text { de las } \\
\text { actividades } \\
\text { educativas }\end{array}$ \\
\hline $\begin{array}{l}\text { Madre } \\
\text { empleada }\end{array}$ & $9.55^{* * *}(4.84)$ & $1.58^{* * *}(.17)$ & $1.44^{* * *}(.16)$ & $1.49^{* * *}(.16)$ & $1.17(.13)$ \\
\hline $\begin{array}{l}\text { Padre } \\
\text { empleado }\end{array}$ & $9.67^{* * *}(3.99)$ & $1.04(.15)$ & $.85(.12)$ & $1.05(.15)$ & $1.29(.22)$ \\
\hline $\begin{array}{l}\text { Madre } \\
\text { teletrabajó }\end{array}$ & $1.69^{* * *}(.20)$ & $1.13(.12)$ & $1.36^{* *}(.14)$ & $1.01(.10)$ & $.85(.10)$ \\
\hline $\begin{array}{l}\text { Pareja } \\
\text { teletrabajó }\end{array}$ & $1.21^{* *}(.07)$ & $.96(.05)$ & $1.01(.06)$ & $1.02(.06)$ & $1.00(.61)$ \\
\hline $\begin{array}{l}\text { Madre con } \\
\text { flexibilidad } \\
\text { horaria }\end{array}$ & $.80 *(.09)$ & $.76^{* *}(.70)$ & $.75^{* *}(.07)$ & $1.07(.10)$ & $1.05(.11)$ \\
\hline $\begin{array}{l}\text { Padre con } \\
\text { flexibilidad } \\
\text { horaria }\end{array}$ & $1.12^{*}(.05)$ & $1.08^{*}(.04)$ & $1.15^{* * *}(.05)$ & $1.07(.04)$ & $1.10^{*}(.05)$ \\
\hline $\begin{array}{l}\text { Madre con } \\
\text { estudios } \\
\text { universitarios }\end{array}$ & $.86(.25)$ & $1.02(.24)$ & $1.02(0.25)$ & $.84(.20)$ & $.93(.24)$ \\
\hline $\begin{array}{l}\text { Madre en } \\
\text { ocupación } \\
\text { de carácter } \\
\text { directivo o } \\
\text { profesional } \\
\text { (CIOU-08 } 1 \\
\text { o 2) }\end{array}$ & $1.57^{*}(.29)$ & $.96(.17)$ & $1.06(.19)$ & $.99(.18)$ & $1.15(.23)$ \\
\hline $\begin{array}{l}\text { Más de un } \\
\text { menor en el } \\
\text { hogar }\end{array}$ & $1.01(.17)$ & $.97(.14)$ & $1.02(.15)$ & $.99(.15)$ & $.68^{*}(.12)$ \\
\hline $\begin{array}{l}\text { Presencia de } \\
\text { menores de } \\
6 \text { años en el } \\
\text { hogar }\end{array}$ & $.71^{*}(.12)$ & $1.12(.17)$ & $.91(.14)$ & $.68^{*}(.10)$ & $1.21(.20)$ \\
\hline Constante & $\begin{array}{c}4.07 \mathrm{e}-10^{* * *} \\
(1.18 \mathrm{e}-09)\end{array}$ & $.15^{*}(.12)$ & $.31(.26)$ & $.16^{*}(.13)$ & $.09^{*}(.08)$ \\
\hline Pseudo R2 & .23 & .003 & .02 & .03 & .01 \\
\hline
\end{tabular}

Fuente: elaboración propia. 
tareas de cuidado. En lo que respecta a las actividades lúdicas, solo el empleo de la madre muestra una asociación significativa (y positiva) con una distribución igualitaria de las horas durante el confinamiento. En el caso de las tareas educativas, únicamente la flexibilidad horaria del padre se asoció con una mayor probabilidad de distribución igualitaria durante el confinamiento.

\section{CONCLUSIONES}

Este estudio ha analizado los patrones de división del trabajo en el seno de las familias con hijos menores a cargo durante el confinamiento por COVID-19, prestando especial atención a la posible importancia del acceso al teletrabajo y/o flexibilidad horaria por parte de ambos progenitores para el establecimiento de un reparto de tareas corresponsable. Se ha partido de la perspectiva teórica de la disponibilidad de tiempo para examinar el impacto potencial de ambos factores como facilitadores de una distribución igualitaria de los tiempos de trabajo y cuidado entre ambos progenitores. Por otro lado, se ha considerado también, a partir de las teorías sobre los recursos relativos y las normas de género, un posible efecto del nivel educativo y del tipo de ocupación de la madre en el resultado de las negociaciones en el seno de las parejas. El objetivo último ha sido detectar una posible brecha, en términos de desigualdad de género, entre parejas con condiciones laborales potencialmente favorecedoras de divisiones del trabajo corresponsables y aquellas que durante el confinamiento no partieron de una situación tan ventajosa.

Los resultados indican, en línea con las expectativas teóricas formuladas, que el primer grupo de familias sí se diferenció del segundo en cuanto a las desigualdades de género establecidas en los hogares durante la emergencia sanitaria por motivos aparentemente relacionados con la disponibilidad de tiempo. Si bien es cierto que en ambos casos se observa una brecha entre hombres y mujeres respecto al tiempo dedicado a cada una de las tareas analizadas, esta fue, en términos absolutos y relativos, menor en las parejas con acceso conjunto al teletrabajo y la flexibilidad. En más de la mitad de estas familias, asimismo, se establecieron divisiones corresponsables del trabajo remunerado, las tareas domésticas, los cuidados, y las actividades lúdicas; situación que contrasta visiblemente con la del segundo grupo, en el que predominaron las distribuciones del tiempo tradicionales. Estos hallazgos sugieren que, de perpetuarse estas tendencias durante el curso de la pandemia, podríamos encontrarnos ante la ampliación de una brecha social basada en las condiciones laborales y el acceso a las medidas de conciliación, ya que la distribución corresponsable del trabajo - especialmente del no remunerado- es un prerrequisito para el mantenimiento de un modelo igualitario de doble 
ingreso que proteja efectivamente a las familias con menores del riesgo de pobreza y vulnerabilidad social. Las características laborales que se asociaron de forma más evidente con una mayor probabilidad de alcanzar arreglos corresponsables variaron según la actividad examinada. En la muestra analizada, el empleo de la madre resultó fundamental para el equilibrio de género en la dedicación doméstica, de cuidado y de juego; el teletrabajo materno para la distribución equitativa del trabajo remunerado, y la flexibilidad horaria del padre para su implicación en la atención educativa. El nivel educativo y la posición ocupacional de las madres no se revelan en este análisis como factores significativos en términos generales, aunque el último factor sí parece haber favorecido divisiones más igualitarias del trabajo remunerado.

Estos resultados contribuyen a la perspectiva teórica basada en la disponibilidad de tiempo de los miembros de la pareja evidenciando la importancia de la disponibilidad conjunta. En un contexto de elevadas demandas domésticas, que ambos progenitores dispongan simultáneamente de condiciones que faciliten la compatibilización de roles como trabajadores y cuidadores (en este caso, teletrabajo) y/o la alternancia en los mismos (flexibilidad) parece ser un factor importante para maximizar las probabilidades de división corresponsable del trabajo. Las investigaciones futuras en este ámbito, más allá de poner el foco en las horas de trabajo de cada miembro de la pareja como ha sido lo habitual en la literatura previa, deberían incrementar la atención a estas dimensiones más cualitativas de los tiempos de trabajo. En particular, es fundamental explorar su relevancia en diferentes contextos y analizar de qué maneras concretas las condiciones de trabajo de la madre y el padre se complementan e interactúan para favorecer o dificultar una distribución equitativa de tareas y responsabilidades. Los hallazgos obtenidos en este estudio también sugieren que la importancia de los recursos relativos y los valores de género individuales para la división del trabajo doméstico pueden pasar a un segundo plano, en determinados casos, en contextos familiares en los que los que la conciliación corresponsable se ve facilitada o impedida por factores estructurales relacionados con las condiciones laborales. Con todo, más allá de las diferencias observadas entre familias en cuanto a las posibilidades de establecer estrategias corresponsables, es preciso destacar la sobrecarga de trabajo asumida en todos los tipos de hogar por las madres. A pesar de la evolución hacia un modelo de división del trabajo más igualitario observada en una proporción aún minoritaria de familias, todo apunta a que han sido las mujeres quienes, una vez más, han absorbido en mayor medida el impacto de la pandemia, esta vez en términos de incremento de las exigencias domésticas. Este fenómeno pone de manifiesto que, a nivel agregado, la persistencia de normas sociales tradicionales en la sociedad continúa dificultando una corresponsabilidad plena en los hogares, incluso en aquellos donde se dan circunstancias más favorables a su consecución. 
Por último, es fundamental recordar las limitaciones metodológicas de este estudio en cuanto a posibilidades de generalización, ya que está basado en una encuesta no representativa y con diversos sesgos potenciales derivados de su administración online. Las conclusiones extraídas de este trabajo deberían ser sometidas a análisis posteriores a partir de fuentes de datos representativas —algunas de ellas ya desarrolladas durante la pandemia en España y otros países de nuestro entorno ${ }^{7}$ - con el fin de contrastar su validez.

\section{Bibliografía}

Aassve, A., Fuochi, G., y Mencarini, L. (2014). Desperate housework: Relative resources, time availability, economic dependency, and gender ideology across Europe. Journal of Family Issues, 35 (8), 1000-1022. Disponible en: https://doi. org/10.1177/0192513X14522248.

Abril, P., Jurado Guerrero, T. y Monferrer, J. M. (2015). Paternidades en construcción. En M. J. González y T. Jurado-Guerrero (comps.). Padres y madres corresponsables: una utopía real (pp. 100-193). Madrid: Los Libros de la Catarata.

Ahrendt, D. et al. (2020). Living, working and COVID-19. First findings, April 2020. Eurofound. Disponible en: https://bit.ly/3mTb2pQ.

Bittman, M., England, P., Sayer, L., Folbre, N., y Matheson, G. (2003). When does gender trump money? Bargaining and time in household work. American Journal of sociology, 109 (1), 186-214. Disponible en: https://doi.org/10.1086/378341.

Brega Baytelman, C. y González Pírez, M. (2020). Coronavirus, conciliación y sesgo de género. Agenda Pública [blog] 19-03-2020. Disponible en: https://bit. ly/3oWTWJe.

Brines, J. (1993). The exchange value of housework. Rationality and society, 5 (3), 302-340.

Carta, G., García, J. R. y Ulloa, C. A. (2020). España: la EPA del 1T20 confirma un impacto notable de la COVID-19 en el mercado laboral. BBVA Research. Disponible en: https://bit.ly/2JBt3uj.

Chung, H. y Van der Lippe, T. (2020). Flexible working, work-life balance, and gender equality: Introduction. Social Indicators Research, 151, 365-381. Disponible en: https://doi.org/10.1007/s11205-018-2025-x.

Coverman, S. (1985). Explaining husbands' participation in domestic labor. Sociological quarterly, 26 (1), 81-97.

Craig, L. y Mullan, K. (2011). How mothers and fathers share childcare: A cross-national time-use comparison. American Sociological Review, 76 (6), 834-861. Disponible en: https://doi.org/10.1177/0003122411427673.

7 Véase Farré et al. (2020). 
Doménech, R. (2020). Reconstruir el mercado de trabajo tras la COVID-19. Análisis regional España. BBVA Research. Disponible en: https://bit.ly/2I65Yzx.

Dominguez-Folgueras, M., Jurado-Guerrero, T. y Botía-Morillas, C. (2018). Against the odds? Keeping a nontraditional division of domestic work after first parenthood in Spain. Journal of Family Issues, 39 (7), 1855-1879. Disponible en: https://doi.org/10.1177/0192513X17729399.

Esping-Andersen, G. (2009). The incomplete revolution: Adapting welfare states to women's new roles. Cambridge: Polity Press.

Esping-Andersen, G., Boertien, D., Bonke, J. y Gracia, P. (2013). Couple specialization in multiple equilibria. European Sociological Review, 29 (6), 1280-1294. Disponible en: https://doi.org/10.1093/esr/jct004.

Farré, L. y Vella, F. (2013). The intergenerational transmission of gender role attitudes and its implications for female labour force participation. Economica, 80 (318), 219-247. Disponible en: https://doi.org/10.1111/ecca.12008.

Farré, L., Fawaz, Y., González, L. y Graves, J. (2020). How the COVID-19 Lockdown affected Gender Inequality in Paid and Unpaid Work in Spain. IZA Institute of Labor Economics, 13434. Disponible en: https://bit.ly/2I693j5.

Gershuny, J., Bittman, M. y Brice, J. (2005). Exit, voice, and suffering: Do couples adapt to changing employment patterns? Journal of marriage and family, 67 (3), 656-665. Disponible en: https://doi.org/10.1111/j.1741-3737.2005.00160.x.

Giovanis, E. (2018). Are Women Happier When Their Spouse is Teleworker? Journal of Happiness Studies, 19 (3), 719-754. Disponible en: https://doi.org/10.1007/ s10902-017-9847-0.

González, M. J. y Jurado Guerrero, T. (2009). ¿Cuándo se implican los hombres en las tareas domésticas? Un análisis de la Encuesta de Empleo del Tiempo. Panorama Social, 10, 65-81.

González, M. J. y Jurado Guerrero, T. (comps.) (2015). Padres y madres corresponsables: una utopía real. Madrid: Los Libros de la Catarata.

González, M. J., Lapuerta, I., Martín-García, T. y Seiz, M. (2018). Anticipating and practicing fatherhood in Spain. En R. Musumecy y A. Santero (comps.). Fathers, childcare and work: Cultures, practices and policies (pp. 17-44). Bingley: Emerald Publishing Limited. Disponible en: https://doi.org/10.1108/S1530353520180000012002.

Gracia, P. (2014). Fathers' child care involvement and children's age in Spain: A time use study on differences by education and mothers' employment. European Sociological Review, 30 (2), 137-150. Disponible en: https://doi.org/10.1093/esr/jcu037.

Gracia, P. y Esping-Andersen, G. (2015). Fathers' child care time and mothers' paid work: A cross-national study of Denmark, Spain, and the United Kingdom. Family Science, 6 (1), 270-281. Disponible en: https://doi.org/10.1080/19424 620.2015 .1082336$.

Gracia, P. y Kalmijn, M. (2016). Parents' family time and work schedules: The split-shift schedule in Spain. Journal of Marriage and Family, 78 (2), 401-415. Disponible en: https:/doi.org/10.1111/jomf.12270. 
Greenstein, T. N. (2000). Economic dependence, gender, and the division of labor in the home: A replication and extension. Journal of Marriage and Family, 62 (2), 322-335. Disponible en: https://doi.org/10.1111/j.1741-3737.2000.00322.x.

Grunow, D. y Evertsson, M. (comp.) (2019). New Parents in Europe. Cheltenham: Edward Elgar Publishing. Disponible en: https://doi.org/10.4337/9781788972970.

Gutiérrez-Domènech, M. (2010). Parental employment and time with children in Spain. Review of Economics of the Household, 8 (3), 371-391. Disponible en: https://doi.org/10.1007/s11150-010-9096-z.

Hiller, D. V. (1984). Power dependence and division of family work. Sex Roles, 10 (11-12), 1003-1019. Disponible en: https://doi.org/10.1007/BF00288521.

Hook, J. L. y Wolfe, C. M. (2012). New fathers? Residential fathers' time with children in four countries. Journal of Family Issues, 33 (4), 415-450. Disponible en: https://doi.org/10.1177/0192513X11425779.

Hupkau, C. y Victoria, C. (2020). COVID-19 y desigualdad de género en España. Madrid: EsadeEcPol.

Instituto Nacional de Estadística (INE) (2020). El teletrabajo en España y la UE antes dla COVID-19. Cifras INE, Boletín Informativo del Instituto Nacional de Estadistica. Disponible en: https://bit.ly/38aeubq.

Lyttelton, T., Zang, E. y Musick, K. (2020). Gender Differences in Telecommuting and Implications for Inequality at Home and Work. Disponible en: https://doi. org/10.31235/osf.io/tdf8c.

Lundberg, S. y Pollak, R. A. (1996). Bargaining and distribution in marriage. Journal of Economic Perspectives, 10 (4), 139-158. Disponible en: https://doi. org/10.1257/jep.10.4.139.

Nock, S. L. (2001). The marriages of equally dependent spouses. Journal of Family Issues, 22 (6), 755-775. Disponible en: https://doi.org/10.1177/019251301022006005.

Noonan, M. (2013). The impact of social policy on the gendered division of housework. Journal of Family Theory and Review, 5 (2), 124-134. Disponible en: https://doi.org/10.1111/jftr.12008.

Palomino, J. C., Rodríguez, J. G. y Sebastián, R. (2020). Teletrabajo en España, ¿estamos preparados para el distanciamiento? Nada es Gratis [blog.], 08-05-2020. Disponible en: https://bit.ly/3er9HDx.

Presser, H. B. (1994). Employment schedules among dual-earner spouses and the division of household labor by gender. American Sociological Review, 59 (3), 348-364. Disponible en: https://doi.org/10.2307/2095938.

Seiz, M., González, M. J., Jurado-Guerrero, T., Lapuerta, I. y Martín-García, T. (2019). Non-normative couples in Spain: mothers' career commitment, fathers' work arrangements, and egalitarian ideologies. En D. Grunow y M. Evertsson (comps.). New Parents in Europe (pp. 169-186). Cheltenham: Edward Elgar Publishing. Disponible en: https://doi.org/10.4337/9781788972970.00019.

Shelton, B. A. y John, D. (1996). The division of household labor. Annual review of sociology, 22 (1), 299-322. Disponible en: https://doi.org/10.1146/annurev.soc.22.1.299.

West, C., y Zimmerman, D. H. (1987). Doing gender. Gender and Society, 1 (2), 125-151. Disponible en: https://doi.org/10.1177/0891243287001002002. 


\section{APÉNDICE}

Tabla 4. Caracteristicas socioeconómicas de la muestra general de personas encuestadas (familias biparentales o monoparentales con hijos menores de 18 años en el hogar)

\begin{tabular}{|c|c|c|}
\hline & $\begin{array}{c}\text { Mujeres } \\
\text { encuestadas } \\
(\mathrm{N}=1019)\end{array}$ & $\begin{array}{c}\text { Hombres } \\
\text { encuestados } \\
(\mathrm{N}=227)\end{array}$ \\
\hline $\begin{array}{l}\text { Edad } \\
18-24 \text { años } \\
25-34 \text { años } \\
\text { 35-44 años } \\
>45 \text { años } \\
\text { Valores perdidos }\end{array}$ & $\begin{array}{c}0,1 \% \\
10,1 \% \\
67,6 \% \\
22,2 \% \\
-\end{array}$ & $\begin{array}{c}- \\
4,4 \% \\
61,2 \% \\
34,4 \%\end{array}$ \\
\hline $\begin{array}{l}\text { Nivel de estudios } \\
\text { Doctorado } \\
\text { Máster } \\
\text { Licenciatura, diplomatura o grado } \\
\text { Formación profesional de cualquier ciclo } \\
\text { Enseñanza general secundaria de segundo ciclo } \\
\text { Enseñanza general secundaria de primer ciclo } \\
\text { Estudios primarios o sin estudios } \\
\text { Valores perdidos }\end{array}$ & $\begin{array}{c}8,7 \% \\
26,0 \% \\
46,6 \% \\
9,6 \% \\
6,1 \% \\
2,4 \% \\
0,5 \% \\
0,1 \%\end{array}$ & $\begin{array}{c}17,2 \% \\
35,7 \% \\
30,8 \% \\
7,9 \% \\
6,2 \% \\
2,2 \% \\
- \\
-\end{array}$ \\
\hline $\begin{array}{l}\text { Situación laboral } \\
\text { Empleada/o (por cuenta ajena o propia) } \\
\text { Desempleada/o } \\
\text { No empleada/o, otra situación } \\
\text { Ama/o de casa } \\
\text { Valores perdidos }\end{array}$ & $\begin{array}{c}85,3 \% \\
5,9 \% \\
5,0 \% \\
3,7 \% \\
0,1 \%\end{array}$ & $\begin{array}{c}95,7 \% \\
3,1 \% \\
0,4 \% \\
0,4 \% \\
0,4 \%\end{array}$ \\
\hline $\begin{array}{l}\text { Profesión (CIOU-08) } \\
\text { Directoras/es y gerentes } \\
\text { Profesionales técnicos e intelectuales } \\
\text { Técnicas/os y profesionales de nivel medio } \\
\text { Personal de apoyo administrativo } \\
\text { Trabajadoras/es de los servicios y vendedoras/es de comercios } \\
\text { y mercados } \\
\text { Agricultoras/es y trabajadoras/es calificadas/os agropecuarias/ } \\
\text { os, forestales y pesqueras/os } \\
\text { Oficiales, operarias/os y artesanas/os de artes mecánicas y de } \\
\text { otros oficios } \\
\text { Operadoras/es de instalaciones de máquinas y ensamblado- } \\
\text { ras/es } \\
\text { Ocupaciones elementales } \\
\text { Valores perdidos }\end{array}$ & $\begin{array}{l}6,7 \% \\
54,4 \% \\
10,1 \% \\
14,3 \% \\
5,6 \% \\
-\end{array}$ & $\begin{array}{c}7,1 \% \\
64,8 \% \\
12,3 \% \\
6,2 \% \\
2,6 \% \\
0,4 \% \\
\\
2,6 \% \\
0,4 \% \\
0,4 \% \\
3,0 \% \\
\end{array}$ \\
\hline
\end{tabular}


Tabla 4. Caracteristicas socioeconómicas de la muestra general de personas encuestadas (familias biparentales o monoparentales con hijos menores de 18 años en el hogar) (continuación)

\begin{tabular}{|l|c|c|}
\hline & $\begin{array}{c}\text { Mujeres } \\
\text { encuestadas } \\
(\mathrm{N}=1019)\end{array}$ & $\begin{array}{c}\text { Hombres } \\
\text { encuestados } \\
\text { (N=227) }\end{array}$ \\
\hline Convivencia de pareja & $90,8 \%$ & $95,6 \%$ \\
Convive con una pareja & $7,3 \%$ & $2,7 \%$ \\
No convive con una pareja porque no tiene & $1,8 \%$ & $1,3 \%$ \\
No convive con una pareja por otros motivos & $0,1 \%$ & $0,4 \%$ \\
\hline Valores perdidos & & \\
\hline Situación laboral de la pareja (si procede) & $82,2 \%$ & $76,2 \%$ \\
Pareja empleada (por cuenta ajena o propia) & $4,3 \%$ & $8,8 \%$ \\
Pareja desempleada & $3,9 \%$ & $5,3 \%$ \\
Pareja no empleada, otra situación & $0,5 \%$ & $5,3 \%$ \\
Pareja ama de casa & $9,0 \%$ & $4,0 \%$ \\
No procede, porque no convive con una pareja & $0,1 \%$ & $0,4 \%$ \\
Valores perdidos & & \\
\hline Número de hijos presentes en el hogar & $39,9 \%$ & $32,2 \%$ \\
Uno & $49,6 \%$ & $51,1 \%$ \\
Dos & $9,3 \%$ & $14,1 \%$ \\
Tres & $1,2 \%$ & $2,6 \%$ \\
\hline Cuatro o más & & \\
\hline Presencia de hijos menores de 3 años & $32,8 \%$ & $33,5 \%$ \\
Sí & $66,9 \%$ & $66,1 \%$ \\
No & $0,3 \%$ & $0,4 \%$ \\
Valores perdidos & & \\
\hline Presencia de hijos menores de 6 años & $61,7 \%$ & $61,9 \%$ \\
Sí & $37,9 \%$ & $38,1 \%$ \\
No & $0,4 \%$ & - \\
Valores perdidos & & \\
\hline Presencia de hijos mayores de 12 años & $14,4 \%$ & $81,1 \%$ \\
Sí & $85,3 \%$ & $18,5 \%$ \\
No & $0,3 \%$ & 0,4 \\
Valores perdidos & & \\
\hline Presencia de hijos con necesidades especiales & & $0,4 \%$ \\
(discapacidad, enfermedad crónica u otras) & & \\
Sí & & \\
No & & \\
Valores perdidos & & \\
\hline
\end{tabular}

Fuente: elaboración propia. 